\title{
THE PATH FROM BIM TO A 3D INDOOR FRAMEWORK - A REQUIREMENT ANALYSIS
}

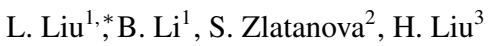 \\ ${ }^{1}$ College of Surveying and GeoInformatics, Tongji University, Shanghai, China - (1.liu, bofeng_li)@tongji.edu.cn \\ ${ }^{2}$ Faculty of Built Environment, UNSW Sydney, Sydney, Australia - s.zlatanova@unsw.edu.au \\ ${ }^{3}$ School of Urban Design, Wuhan University, China - liu.hua@whu.edu.cn
}

\section{Commission IV, WG IV/5}

KEY WORDS: Building Information Modelling, Geographic Information System, 3D Indoor Framework, Spatial Data Model, Requirements Analysis, 3D Spatial Analysis

\begin{abstract}
:
Building Information Modelling is a new technology and tool for researchers in Architecture/Construction/Engineering (ACE) fields, and it is designed to organise the collaboration between different parties and manage all types of information regarding buildings. A standardized data model of BIM named Industry Foundation Classes (IFC) has been proposed to describe building \& construction related information and facilitate the interoperability of building data. As IFC contains plenty of building information, further spatial analysis can be applied in geographical information system (GIS) with these information. Current research has mainly discussed the requirements about data translation from BIM to GIS. However, more specific requirements on the functionalities of a generic 3D indoor framework need to be considered with IFC data. Although this research is in the initial stage, we investigate the use of BIM IFC for the 3D indoor framework, especially on the data translation from IFC to the framework, data management in the framework and IFC data for 3D spatial analysis. Requirements are analysed in four aspects: 1) automation of indoor vector map generation; 2) data management for the framework; 3) environmental analysis of the framework; and 4) safety analysis of the framework. According to these requirements, new modules need to be developed. In the future, based on IFC data we will conduct tests of data transformation, design a data model for the 3D indoor framework and develop a prototype to demonstrate the planned spatial analysis functionalities.
\end{abstract}

\section{INTRODUCTION}

Building information modeling (BIM) is an active new technology and tool in Architecture/Construction/Engineering (ACE) fields, and it is designed to organise the collaboration between different parties and manage all types of building information. Specifically, the standardized data model named Industry Foundation Classes (IFC) is proposed to describe building information and facilitate the interoperability of building data. As IFC data contain abundant indoor spatial information, geographical information system (GIS) can be considered to provide further spatial analysis based on these information. In addition, IFC data contain plenty of structural or constructional information and thus the information need to be refined for GIS.

GIS is designed for mapping, modelling and analysis of spatial data. As IFC contains many building-design features and detailed properties of buildings, it has increasingly been considered an ideal data source about indoor environments and been translated in GIS-related studies such as indoor navigation model generation (Taneja et al., 2016). More specifically, BIM IFC provides a standard data model which can be selectively implemented in GIS platforms for specific purposes such as location-based services ( $\mathrm{Li}$ and $\mathrm{He}$, 2008) (Teo and Cho, 2016), asset management (Boyes et al., 2017) (Kang and Hong, 2015) or hazard simulations (Tang and Kim, 2004). In a building-involved context, facility managers and decision-makers are the key users who need translation of BIM data into GIS platforms.

There are reasons for the translation of BIM data to GIS. Cur-

\footnotetext{
${ }^{*}$ Corresponding author
}

rently three dimensional (3D) GIS is still in development and it needs to incorporate existing data to represent the insides of building. 3D GIS can make use of IFC since IFC is one of the standard formats of building. Moreover, a single IFC data file for a building could be large in size, and it comprehensively stores the accurate state of the building and its elements and accessories. But this file could not reflect building dynamics (i.e., the changing environment). In contrast, GIS provides a concise way to manage dynamic data via geo-database and provides abundant tools for spatial analysis.

Spatial analysis is one of the fundamental characteristics of GIS. For example, spatial statistics supports the analysis of georeferenced data (e.g., spatial interpolation). A recent research presents the noise analysis in the insides of a building and visualise noise influence (Deng et al., 2016b). Similarly, the analysis of other indoor environmental factors such as temperature and humidity can be introduced. Such analyses are based on the translation of IFC data to GIS platforms. In this sense, researchers in ACE fields can also obtain extra knowledge about buildings with spatial analysis in a GIS platform (e.g., evacuation simulation in a building).

Researchers have investigated the requirements regarding translating BIM into GIS. Considering the translation from the IFC data model to a new designed data model for indoor navigation, Isikdag et al. (Isikdag et al., 2013) propose related conceptual requirements on a building model. These requirements aim to preserve semantic information and properties of building, real 3D structural information (e.g., vertical levels), spatial relationships of building elements and their interoperable geometry. In this example, the authors focus on the necessary portion of IFC data to 
meet indoor navigation requirements. Another review paper discusses the interoperability of BIM and GIS (Liu et al., 2017), and it categorises the integration of BIM and GIS into three levels, i.e., data, process and application. It also reviews the data models to preserve the complete information in both IFC and GIS with a generic ontology. For a GIS platform, this review paper mainly shows the requirements on data translation and incorporation of IFC.

However, more specific requirements need to be clarified for GIS applications with IFC data, i.e., how to use the data converted from IFC and what are the analysis functionalities? For these applications, we consider a generic 3D indoor framework which can provide the modelling and analysis functionalites on $3 \mathrm{D}$ objects in indoor environments. Although this research is in the initial stage, this paper aims to reveal the requirements about the $3 \mathrm{D}$ indoor framework. In general, this framework is designed to incorporate 3D indoor modelling with spatial analysis. Thus BIM IFC can support this framework at least in the following aspects: 1) indoor vector maps; 2) data management of the framework; 3 ) indoor environmental analysis; and 4) emergency scenario simulation such as fire proliferation. So far there is no generic solution which can completely address the above topics. In this paper we conduct a requirement analysis focusing on the four aspects, and explain potential application scenarios with examples.

This paper is organised as follows. Section 2 will present a brief background on the relationship between BIM and GIS, which gives the big picture of current GIS applications with IFC data. Section 3 will present a requirement analysis on the four aspects. Subsection 3.1 introduces the need of automatic generation of vector map from IFC data; subsection 3.2 discusses the requirements on data management of the framework; subsection 3.3 presents the need for indoor environmental analysis such as the distribution of noise or temperature; and subsection 3.4 provides the requirements on indoor safety analysis such as fire diffusion. Section 4 concludes this paper with some future work.

\section{BACKGROUND}

So far the most concerned topic is the data interoperability between IFC and other data models in GIS domain. For instance, there are reported studies on mapping IFC to CityGML data (Cheng et al., 2013) (Deng et al., 2016a). CityGML (City Geography Markup Language) is an Open Geospatial Consortium $(O G C)$ standard and it is used as a common information model for geometric and semantic representations of 3D urban objects (Gröger et al., 2012). Cheng et al. (Cheng et al., 2013) discuss the transformation from IFC geometry of Constructive Solid Geometry (CSG) to Boundary Representation (B-Rep) in CityGML. But there is no comprehensive standard to bridge IFC and GIS-related data models, mostly because these standards are developed for different purposes in spite of their correlations.

The overlap between BIM and GIS lays on the 'building' notion. But the integration of BIM and GIS is not required for every building-related application. Specifically, the developing 3D GIS can benefit from the development of BIM technologies. Naturally IFC data are applied to visualisation of building insides (Arslan et al., 2014). Some research regards IFC data as 3D indoor background (Costin and Teizer, 2014) (Arslan et al., 2014). Studies regarding indoor navigation emphasize the $3 \mathrm{D}$ geometry of BIM data and generate accurate indoor maps ( $\mathrm{Li}$ and $\mathrm{He}, 2008)$ (Teo and Cho, 2016) (Wu and Zhang, 2016) (Diakité and Zlatanova, 2018). In this study, the navigation network representing the accessible path network is derived from the 2.5D floor maps extracted from the 3D geometric elements in BIM. Indoor positioning and localisation can also benefit from BIM data. The geometry in BIM data serves as a constraint to improve indoor localisation results. Tomasi et al. (Tomasi et al., 2015) show the analysis functionality for the deployment of positioning sensors in a building.

3D spatial query is a pivotal feature of 3D GIS and it can be applied to the information extracted from BIM data. Boyes $e t$ al. (Boyes et al., 2017) propose a combined use of BIM and GIS for asset management. They aim for location-based queries ("objects in a space" or "the asset data regarding this location"). The authors prefer to convert BIM data to a GIS platform. Kang and Hong (Kang and Hong, 2015) propose a method to extract geometric shapes and element properties from IFC data to GIS in terms of facility management. Irizarry et al. (Irizarry et al., 2013) combine BIM and GIS to support supply chain management of construction suppliers. Fosu et al. (Fosu et al., 2015) suggest to maintain asset database to monitor the energy consumption of buildings.

Liu et al. (Liu et al., 2017) categorise the integration of BIM and GIS into three levels: data level, process level and application level. On the data level, as BIM IFC contains much more details of buildings than the building-related data models in GISdomain, research is normally conducted on the translation from IFC to these GIS-related data models (e.g., CityGML). For such a translation, researchers have to select and simplify the schema of IFC for applications. On the process level, a generic ontology can be created for both BIM and GIS domains. This method is flexible but it is still under development; on the application level, intermediate information is adopted to connect BIM and GIS for a specific problem. However, this categorisation concentrates on the data interoperability but not on different roles of BIM and GIS.

Several review papers stress the analysis functionalities of GIS (Fosu et al., 2015) (Liu et al., 2017). They identify the fact that BIM data provide accurate models to a GIS platform for spatial environmental analysis. Stoter et al. (Stoter et al., 2008) visualise and quantify noise influence around the façade of a building, which is a typical measure in a GIS platform. A further implementation has extended the noise analysis into the insides of a building (Deng et al., 2016b).

As BIM IFC comprehensively defines building elements with their properties and their relationships, it is an ideal data model for indoor dynamics simulation and analysis. In a study about simulations for building design, Tang and Kim (Tang and Kim, 2004) initially discuss different types of simulation for buildings in an analysis platform. These simulation scenarios include energy simulation such as thermal or solar shadow mapping, airflow analysis, the movement of fire and smoke dynamics, occupant evacuation in a 3D environment, indoor light analysis, etc. All these analyses and simulations correspond to physical properties of building elements and their materials. It is noted that these analyses are conducted with a toolkit (Tang and Kim, 2004), which indicates outside analysis plug-in can complement CAD software to enrich analysis functionalities. Similarly, with a toolkit Dimyadi et al. (Dimyadi et al., 2007) generate fire diffusion information based on IFC data. During this procedure, the authors also extract the geometric and topological information of building from the IFC data. 
In general, the active research directions regarding both BIM and GIS include: 1) indoor navigation. Navigation networks can be derived based on the geometry and semantics of IFC ( $\mathrm{Li}$ and $\mathrm{He}$, 2008) (Teo and Cho, 2016); 2) location-based services. It includes indoor localisation and asset/facility management (Boyes et al., 2017) (Kang and Hong, 2015); 3) layout planning. Layout planning can be applied for the computation of the optimal coverage of wireless sensors or lights (Tomasi et al., 2015); 4) environmental analyses, such as energy consumption analysis, shadow analysis (Rafiee et al., 2014), and noise analysis (Stoter et al., 2008) (Deng et al., 2016b); and 5) dynamic factor analyses. Such analyses can be fire/smoke simulation and evacuation analysis (Tang and Kim, 2004).

Regarding the different roles of BIM and GIS, currently the general opinion emphasizes the analysis ability of GIS and accurate building-design features and detailed properties of BIM (Irizarry et al., 2013) (Wu and Zhang, 2016). Barazzetti and Banfi (Barazzetti and Banfi, 2017) indicate BIM software has limited processing ability on geo-spatial data. The authors introduce a practical tool Autodesk Infrawork issued by Autodesk, Inc. to support BIM processes. Autodesk Infrawork can handle both raster (e.g., aerial/satellite images) and vector (e.g., TIN) formats. It also provides a connection to data from ArcGIS (the famous GIS software set of Esri, Inc.) and thus building data can be geo-referenced. Therefore, it is not difficult to load and visualise geo-spatial data for BIM software. But more geo-spatial queries/analyses are required to interpret and comprehend indoor dynamics and phenomena. We argue that the research focus regarding BIM to GIS can be shifted to more practical topics, i.e., to develop and optimise more spatial analysis functionalities which can facilitate the use of BIM information. In the following section, we will present a requirement analysis on a generic 3D indoor framework which aims to integrate 3D models with spatial analysis functionalities.

\section{REQUIREMENTS ANALYSIS}

In this section, we present the requirement analysis about the development of a generic 3D indoor framework on IFC information. These requirements mainly cover four topics: 1) automation of indoor vector map from IFC; 2) data management regarding spatial analysis; 3) environmental analysis based on IFC information; and 4) indoor safety analysis based on IFC information.

\subsection{Indoor Vector Map}

Vector map is a basic input for GIS platforms. As BIM IFC provides accurate $3 \mathrm{D}$ models of building with precise dimensions, it has the potential to be automatically transferred to indoor vector maps. In this case, floor plans (i.e., the vector map) can be derived on each storey according to $C S G$ geometric elements. Many researchers have utilised IFC data to facilitate the automation of indoor vector maps. A major problem with these methods is that they cannot conduct a complete automation since they cannot handle some complicated 3D geometric shapes (e.g., staircase or escalator). For example, a spiral staircase consisting of many steps is non-trivial for GIS data management.

Figure 1 presents an example of indoor maps derived from IFC data. The stair (consisting of solids, see the upper part) are converted to a 3D surface geometry in the 3D indoor framework (see the bottom part). At present the existing methods for navigation network generation always automatically derive flat network in
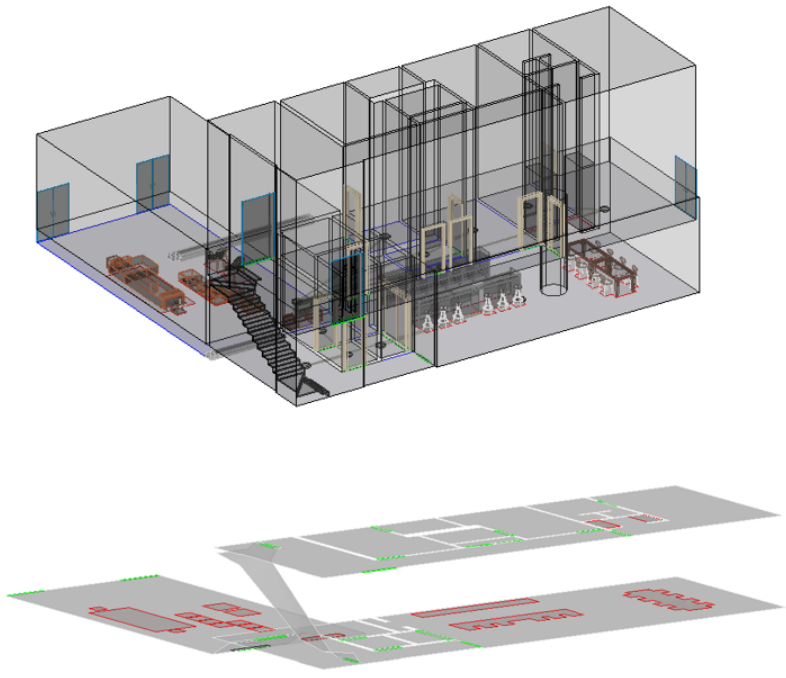

Figure 1. Illustration of indoor vector map generation

single floors, but they either symbolise the vertical passage or manually outline its shape.

Another important issue is the validation of the geometry of IFC models. As IFC models are manually created, it is possible to involve some topological/geometric errors (e.g., overlap). For the purpose of indoor navigation, a recent study has provided a test for geometric data validation of IFC data (Diakité and Zlatanova, 2016). The geometry of IFC need to be checked and validated before indoor maps and navigation networks are derived.

Therefore, the requirements for the generation of indoor vector map are listed as follows:

- Develop a complete automation method to transform 3D geometry in IFC to indoor maps.

- Validate geometric data in IFC for the generic 3D indoor framework.

\subsection{Data Management}

The requirement for indoor data management is to organise geometric and semantic data of building and its properties in a concise and effective way. Considering the functionalities of the $3 \mathrm{D}$ indoor framework, it is not designed to process all kinds of queries about building. We argue that this 3D indoor framework can leave design and construction data to BIM software, but only focuses on the analysis functionality on building with its geospatial context. Based on the current implemented data models in GIS, extension modules can be added to absorb new information types from BIM IFC.

All the data converted from BIM IFC need to be managed in an appropriate way. Many BIM classes are new to GIS and they can be introduced to the design of new GIS data models. Therefore, the corresponding new data models should aim for data storage for different applications. For instance, several classes defined in the domain specific data schemas of IFC can be transformed to the 3D indoor framework, i.e., IfcHvacDomain, IfcPlumbingFireProtectionDomain, IfcElectricalDomain, and IfcBuildingControlsDomain. IfcHvacDomain schema involves the concepts 
in the heating, ventilating and air conditioning (HVAC) domain; IfcPlumbingFireProtectionDomain schema defines concepts related to plumbing and fire protection. It relates to terminal points or hydrants for extinguishing fire, which can support fire simulation; and IfcElectricalDomain schema includes cabled systems for electrical supply, which can be applied to energy consumption analysis. In addition, in the shared element data schemas of IFC, If SharedFacilitiesElements schema provides basic concepts for facilities management (FM); and IfcSharedBldgServiceElements schema relates to property sets of flow and distribution systems, e.g., fluid-flow properties, electrical properties and thermal properties. All these properties relate to indoor environments and they can be stored in the 3D indoor framework for spatial analysis. To keep the integrity and independence of data, a generic data model can be designed for different applications. For example, pipelines can be converted and stored with air-related data (ventilation and pressure), and thus air flow can be analysed and visualised in the 3D indoor framework. Similarly, other building data (e.g., materials) for safety analysis can also be appropriately organised for needed application analysis.

A conceptual mapping of BIM information to the 3D indoor framework is given in Figure 2. Four components of IFC data, geometry, semantics, relationships and properties of building elements, are converted into the 3D indoor framework. The IFC geometry could be converted to different kinds of shapes such as Grid, B-rep, and Octree (see Figure 2). Semantics of building elements and relationships between IFC classes could be transformed to the semantics of the framework. Also, properties are converted to the attributes of geometric shapes in the framework. At last, the geometry, the related semantics and attributes could be managed in Geo-DBMS.

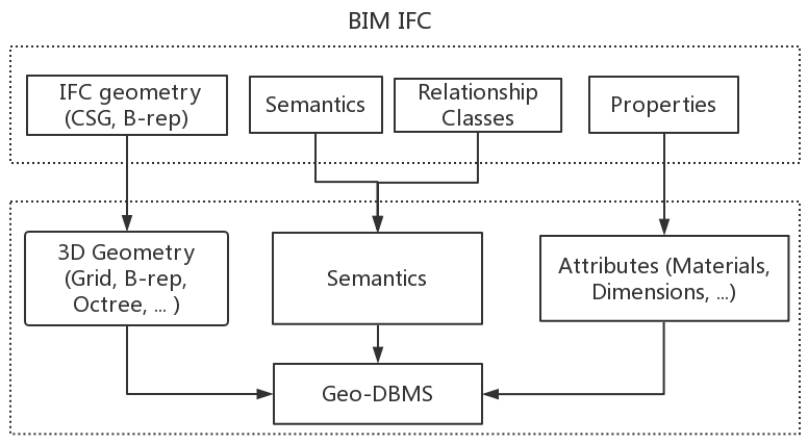

3D Indoor Framework

Figure 2. Conceptual mapping of IFC and the 3D indoor framework

In summary, the requirements on data management of IFC information in the framework are:

- Keep source domain models, and organise them as extension modules to the data model of the generic 3D indoor framework.

- Link four components (geometry, semantics, relationship and property) of IFC to the corresponding sections of the framework.

\subsection{Indoor Environmental Analysis}

3D spatial analysis methods can be integrated into the 3D indoor framework and they could be applied to building information ex- tracted from IFC. IFC is a container of infrastructure data but without analysis functionalities. Thus the 3D indoor framework can make use of spatial statistics and incorporate the data from IFC for indoor environmental analysis. It should provide not only queries about single building elements/facility/location, but also statistics for indoor environmental factors or phenomena. For example, one can analyse the movement of air flow in this framework and detect the section that may involve air quality problem; or can estimate energy consumption in a building with electrical facility information in IFC and indicate the highest consumption group by cluster analysis. Another example is configuration planning for a lighting system. IFC presents accurate lighting devices and their relationships, thus one can analyse the coverage of the lighting system in this framework and generate different solutions according to different criteria.

Compared to the traditional 2D spatial analysis on maps, the 3D indoor framework needs generic data structures to extend some analysis functions such as overlay. Overlay analysis puts together information on different 2D layers and thus provides an overview on the attributes of map elements. Overlay analysis can also be useful for 3D indoor environments. For example, the distribution of temperature and airflow can be overlaid to analyse the cooling status in a building. However, in this 3D indoor framework different attributes of a 3D space can not easily be overlaid since the space is three dimensional. Therefore, an appropriate generic data structure (e.g., voxels) could be used for such spatial analysis functionalities.

Compared to the IFC schemas IfcElectricalDomain, IfcBuildingControlsDomain and IfcHvacDomain, the 3D indoor framework needs a new module to receive and store real-time data of different kinds of indoor sensors (e.g., temperature, humidity, noise, and wireless signal strength). In this case, the module can be used to monitor the status of different environmental factors. Based on the collected data, the corresponding distribution of a factor regarding the whole building can be mapped in this framework on demand. For example, the distribution of wireless signal strength can be used for indoor localisation.

The requirements on indoor environmental analysis in the 3D indoor framework are presented as follows:

- Use a generic data structure for spatial statistics in this 3D indoor framework.

- To conduct indoor environmental analysis, design and implement 3D spatial analysis methods for the framework.

- Design a real-time data module for the framework.

\subsection{Indoor Safety}

The generic 3D indoor framework needs to consider the computation and simulation of indoor emergency cases such as fire, smoke and pedestrian flow. Fire and smoke represent hazards in emergency situations and they change over time; pedestrian flow needs to be planned as occupant evacuation. IFC provides many structure-related information including the materials of building components and their connections. The materials of building elements can be linked to their flammability. Besides, IFC also provides fire protection information. By utilizing this information, 
this framework should perform the simulation to estimate fire development and evaluate the corresponding hazard level. Following that, the framework should provide evacuation plan based on the fire/smoke diffusion simulation.

Although there are studies on fire/smoke simulation and evacuation (Dimyadi et al., 2007) (Xiong et al., 2017), the simulation functionalities can also be introduced to the $3 \mathrm{D}$ indoor framework. The core of such simulations is the mathematical model of the dynamics. A new module could be developed for the 3D indoor framework to adopt mathematical models of fire/smoke movement, which is similar to the previous methods for indoor dynamic simulations (Dimyadi et al., 2007) (Jevtić, 2015). The module can be designed with an interface to edit these models. The simulation results of different mathematical models can be compared to provide relatively objective damage evaluation. In addition, data in the IFC schema IfcPlumbingFireProtectionDomain contain hydrants for extinguishing fire. The computation of fire development can take the function of these hydrants into account. In this case, the 3D indoor framework can provide computation and visualisation of the development of a certain hazard factor (fire or smoke). It can also estimate and predict the impacted spaces and objects along with time.

As mentioned before, real-time data collection needs to be considered in this 3D indoor framework. In this case, first responders can make use of all types of sensors including smoke detector, temperature indicator, video monitor and indoor positioning device. These real-time data can support fire/smoke development estimation and evacuation planning. Evacuation planning can be regarded as an optimisation problem which optimises the computation with multiple criteria. These criteria include the least total evacuation time, the least congestion and other customized criteria. The data of video monitor and positioning device can be used to estimate evacuee numbers and their movement. An interface needs to be designed for users to input criteria to obtain different evacuation planning results.

The requirements on indoor safety analysis in the $3 \mathrm{D}$ indoor framework are presented as follows:

- Incorporate sensor data for fire/smoke simulation and evacuation planning in the generic 3D indoor framework.

- Incorporate hazard simulation functionality in the framework, and design an interface to select/input mathematical models of fire/smoke movement.

- Incorporate evacuation planning functionality in the framework, and design an interface to input evacuation criteria.

\section{CONCLUSIONS}

As BIM is adopted for information modelling for researchers in AEC fields, it concentrates on the accurate descriptions on building system, elements and accessories. These information can be refined for spatial analysis of a generic 3D indoor framework. This framework aims for the integration of 3D modelling and spatial analysis. The motivation behind this work is to facilitate intelligent translation from BIM to the framework and to apply more spatial analysis functionalities on the extracted 3D geometries with semantic data of BIM.

Though this research is in the early stage, we present an analysis on the requirements about possible development for the generic
3D indoor framework with BIM IFC. More specifically, we analyse the requirements in four aspects: 1) automation of indoor vector map generation; 2) data management for the 3D indoor framework; 3) environmental analysis of the framework; and 4) safety analysis of the framework. Accordingly, new modules need to be developed for this framework, and these requirements are summarised as follows:

- Automation method to transform 3D geometry in IFC; and validation of the geometry.

- Translation of IFC geometry, semantics, relationship and property to the framework; and data storage for different domains.

- A generic data structure for spatial statistics in 3D environments; implementation of 3D spatial analysis in the framework; and a real-time data module of the framework.

- Sensor data for fire/smoke simulation and evacuation planning; hazard simulation functionality and an interface for mathematical models input; evacuation planning functionality and an interface for evacuation criteria input.

In the future, we will conduct the testing of data transformation from IFC to the 3D indoor framework, design a data model for the framework and implement it in Geo-DBMS. Based on the data model, we will continue to develop the functionalities of spatial analysis for the $3 \mathrm{D}$ indoor framework and then test them with real-time data. After that, a demonstration prototype will be developed and presented.

\section{ACKNOWLEDGEMENTS}

This study was supported by National Natural Science Foundation of China (41574023, 41622401 and 41471324), Scientific and Technological Innovation Plan from Shanghai Science and Technology Committee (17511109501, 17DZ1100802, and 17DZ1100902), and National Key Research and Development Program of China (2016YFB0501802).

\section{REFERENCES}

Arslan, M., Riaz, Z., Kiani, A. K. and Azhar, S., 2014. Real-time environmental monitoring, visualization and notification system for construction h\&s management. Journal of Information Technology in Construction (ITcon) 19(4), pp. 72-91.

Barazzetti, L. and Banfi, F., 2017. Bim and gis: when parametric modeling meets geospatial data. ISPRS Annals of the Photogrammetry, Remote Sensing and Spatial Information Sciences 4(5W1), pp. 1-8.

Boyes, G., Ellul, C. and Irwin, D., 2017. Exploring bim for operational integrated asset management-a preliminary study utilising real-world infrastructure data. In: ISPRS Annals of the Photogrammetry, Remote Sensing and Spatial Information Sciences, Vol. 4number 4W5, pp. 49-56.

Cheng, J., Deng, Y. and Du, Q., 2013. Mapping between bim models and 3d gis city models of different levels of detail. In: 13th international conference on construction applications of virtual reality, London, pp. 30-31.

Costin, A. and Teizer, J., 2014. Utilizing bim for real-time visualization and indoor localization of resources. In: Computing in Civil and Building Engineering (2014), pp. 649-656. 
Deng, Y., Cheng, J. C. and Anumba, C., 2016a. Mapping between bim and $3 \mathrm{~d}$ gis in different levels of detail using schema mediation and instance comparison. Automation in Construction 67, pp. 1-21.

Deng, Y., Cheng, J. C. P. and Anumba, C., 2016b. A framework for $3 \mathrm{~d}$ traffic noise mapping using data from bim and gis integration. Structure \& Infrastructure Engineering 12(10), pp. 12671280 .

Diakité, A. A. and Zlatanova, S., 2016. Valid space description in bim for $3 \mathrm{~d}$ indoor navigation. International Journal of 3-D Information Modeling (IJ3DIM) 5(3), pp. 1-17.

Diakité, A. A. and Zlatanova, S., 2018. Spatial subdivision of complex indoor environments for $3 \mathrm{~d}$ indoor navigation. International Journal of Geographical Information Science 32(2), pp. 213-235.

Dimyadi, J. A., Spearpoint, M. and Amor, R., 2007. Generating fire dynamics simulator geometrical input using an ifc-based building information model. Electronic Journal of Information Technology in Construction 12, pp. 443-457.

Fosu, R., Suprabhas, K., Rathore, Z. and Cory, C., 2015. Integration of building information modeling (BIM) and geographic information systems (GIS)-a literature review and future needs. In: Proceedings of the 32nd CIB W78 Conference, Eindhoven, The Netherlands.

Gröger, G., Kolbe, T., Nagel, C. and Häfele, K., 2012. OGC City Geography Markup Language (CityGML) Encoding Standard.

Irizarry, J., Karan, E. P. and Jalaei, F., 2013. Integrating bim and gis to improve the visual monitoring of construction supply chain management. Automation in Construction 31(31), pp. 241-254.

Isikdag, U., Zlatanova, S. and Underwood, J., 2013. A bimoriented model for supporting indoor navigation requirements. Computers, Environment and Urban Systems 41, pp. 112-123.

Jevtić, R., 2015. The fire simulation as a safety advantage in fire prediction and fire protection. Safety Engineering 5(1), pp. 2128.

Kang, T. W. and Hong, C. H., 2015. A study on software architecture for effective bim/gis-based facility management data integration. Automation in Construction 54, pp. 25 - 38.

Li, Y. and He, Z., 2008. 3d indoor navigation : a framework of combining bim with $3 \mathrm{~d}$ gis. In: Proceedings of 44th The International Society of City and Regional Planners (ISOCARP) Congress 2008.

Liu, X., Wang, X., Wright, G., Cheng, J. C. P., Li, X. and Liu, R., 2017. A state-of-the-art review on the integration of building information modeling (bim) and geographic information system (gis). ISPRS International Journal of Geo-Information.

Rafiee, A., Dias, E., Fruijtier, S. and Scholten, H., 2014. From bim to geo-analysis: View coverage and shadow analysis by bim/gis integration. Procedia Environmental Sciences 22, pp. 397-402.

Stoter, J., De Kluijver, H. and Kurakula, V., 2008. 3d noise mapping in urban areas. International Journal of Geographical Information Science 22(8), pp. 907-924.

Taneja, S., Akinci, B., Garrett, J. H. and Soibelman, L., 2016. Algorithms for automated generation of navigation models from building information models to support indoor map-matching. Automation in Construction 61, pp. 24-41.
Tang, D. and Kim, J., 2004. Simulation support for sustainable design of buildings. In: Proc. of CTBUH Conference, Vol. 1013, pp. 208-213.

Teo, T.-A. and Cho, K.-H., 2016. Bim-oriented indoor network model for indoor and outdoor combined route planning. $A d$ vanced Engineering Informatics 30(3), pp. 268-282.

Tomasi, R., Sottile, F., Pastrone, C., Mozumdar, M. M., Osello, A. and Lavagno, L., 2015. Leveraging bim interoperability for uwb-based wsn planning. IEEE Sensors Journal 15(10), pp. 5988-5996.

Wu, B. and Zhang, S., 2016. Integration of gis and bim for indoor geovisual analytics. International Archives of the Photogrammetry, Remote Sensing \& Spatial Information Sciences.

Xiong, Q., Zhu, Q., Du, Z., Zhu, X., Zhang, Y., Niu, L., Li, Y. and Zhou, Y., 2017. A dynamic indoor field model for emergency evacuation simulation. ISPRS International Journal of Geo-Information. 